\title{
Investigation of leakage holes created by four needle types used for closure of canine enterotomies
}

\author{
Konstantinos Mitsou ${ }^{1}$, Lysimachos G. Papazoglou ${ }^{1, *}$, Ioannis Savvas ${ }^{1}$ and Emmanouil Tzimtzimis ${ }^{2}$ \\ ${ }^{I}$ Department of Clinical Sciences, School of Veterinary Medicine, Aristotle University of Thessaloniki, Thessaloniki, \\ Greece \\ ${ }^{2}$ Vale Referrals, Stinchcombe, Dursley, Gloucestershire, UK
}

\begin{abstract}
The creation of leakage holes in the intestinal wall by four types of swaged-on needles used to close enterotomy incisions in canine cadavers was studied. Twenty-four enterotomies were performed in $10 \mathrm{~cm}$ jejunal sections obtained from five dogs following euthanasia. After placement of Doyen intestinal forceps to the ends of each section, a $3 \mathrm{~cm}$ antimesenteric incision was performed and closed using 4-0 polydioxanone suture armed in a swaged-on needle in a simple interrupted pattern. One group served as control, with no enterotomies performed. The four groups that we used consisted of six sections each: group $\mathrm{CC}$ was closed with a polydioxanone suture armed in a conventional cutting needle, group RC was closed with a reversed cutting needle, group TPP was closed with a taper point plus needle, and group TC was closed with a taper cutting needle. Leak testing was performed by infusion of $13.5 \mathrm{~mL}$ methylene blue solution into the intestinal lumen. Significant differences between leakage and non-leakage sections of group CC were detected $(P=.027)$. No statistical differences were detected among other groups. Conventional cutting needles seem to create leaking holes in cadaveric healthy jejunum during needle passage for closing an enterotomy incision.

Keywords: Dog, Enterotomy, Leakage, Needle holes, Surgical needles.
\end{abstract}

\section{Introduction}

Enterotomy is a commonly performed surgical procedure in small animals. Indications for an enterotomy procedure include removal of intestinal foreign bodies or biopsy (Fossum and Hedlund, 2003). Enterotomy closure is achieved by simple interrupted or continuous sutures armed in swaged-on needles that are necessary for the placement of sutures through the intestinal wall without disruption of tissue architecture or by the use of skin staples (Weisman et al., 1999; Coolman et al., 2000; Fossum and Hedlund, 2003; Ellison, 2014; Schwartz and Coolman, 2018). However, creation of suture holes is achieved by suturing needles that must pass through intestinal tissue away from the incision (Fossum and Hedlund, 2003; Thomson, 2007). The larger the created holes are, the greater is the possible leakage of intestinal contents, and bleeding through the needle holes, leading to high morbidity and mortality associated with septic peritonitis (Thomson, 2007).

The needle types used in intestinal surgery include taper, taper cutting, or reverse cutting needles (Weisman et al., 1999; Baines, 2000; Coolman et al., 2000; Fossum and Hedlund, 2003; Shales et al., 2005; Ellison, 2014). Conventional cutting needles are not recommended because of significant trauma and the larger hole they create to the intestinal wall during needle passage (Trier, 1979; Bellenger, 1982; Dunn,
2005; Schmiedt, 2012). To the authors' knowledge, no studies in the literature investigate the effect of needle type on needle hole creation and subsequent leakage following needle passage through the intestinal wall during intestinal surgery.

The aim of the present study was to compare four types of needles used to close enterotomy incisions in canine cadavers in relation to the creation of leakage holes in the intestinal wall, as tested by infusion of methylene blue solution into the intestinal lumen. Our hypothesis was that taper, taper cutting, or reverse cutting needles are secure options for performing a successful enterotomy closure, whereas conventional cutting needles are not.

\section{Materials and Methods}

Healthy jejunum was harvested from cadavers of five client-owned dogs euthanized for reasons unrelated to digestive tract pathology after owners' consent was given. Euthanasia was performed by administering an overdose of propofol. The jejunum was harvested immediately after euthanasia and cut into $80 \mathrm{~cm}$ segments; intestinal contents were gently removed by saline infused into the lumen, placed into $0.9 \%$ sodium chloride solution. All segments were stored at $4{ }^{\circ} \mathrm{C}$ until testing, which was performed within 3 hours following euthanasia. The jejunal segments were removed from the saline solution and cut into sections of $10 \mathrm{~cm}$ for enterotomies (Saile et al., 2010).

*Corresponding Author: Lysimachos G. Papazoglou. Department of Clinical Sciences, Faculty of Veterinary Medicine, 
The intestinal constructs were randomly allocated (Research Randomizer, G. C. Urbaniak and S. Plous, USA) into five groups of six sections each. One group served as negative control, with no enterotomy performed. Following placement of Doyen intestinal forceps to the ends of each section, a $3 \mathrm{~cm}$ antimesenteric incision was performed using a \# 15 blade and scissors, and closure was performed with 4-0 polydioxanone suture armed in a swaged-on needle in a simple interrupted pattern. A new needle was used for each section that was sutured. For each enterotomy, 10 simple interrupted sutures were placed 2-3 mm apart and $3 \mathrm{~mm}$ away from the incision edges. Four square knots were used to secure each suture. Group CC was closed with a polydioxanone suture armed in a conventional cutting needle (PDS II, PC-3 cutting 16 $\mathrm{mm} 3 / 8 \mathrm{c}$, Ethicon, USA), group RC was closed with a reversed cutting needle (PDS II, FS-2 reverse cutting

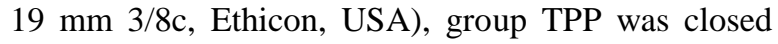
with a taper point plus needle (PDS II, RB-1 plus taper

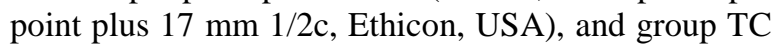
was closed with a taper cutting needle (PDS II, V-5

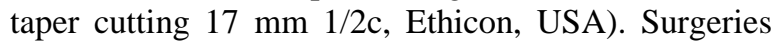
were performed by the same clinician (KM).

Leak testing was performed immediately following closure. The constructs were tested in random order. The clinician (LGP) that performed the testing was not aware of the type of needle used. For testing, methylene blue solution was prepared (1 part of methylene blue: 100 parts of $0.9 \%$ sodium chloride solution) and infused into each segment by using a $20 \mathrm{~mL}$ syringe attached to a $23 \mathrm{G}$ butterfly needle that was inserted into the lumen $2-3 \mathrm{~cm}$ away of the incision at the antimesenteric side. The volume of methylene blue infused in each section was $13.5 \mathrm{~mL}$. Leakage was defined by observation of dye- containing solution around one or more suture holes. Results from the leak testing were analyzed with SPSS software. Pearson chisquare test was used to compare the differences among groups for the presence of suture hole leakage. Significance was set at values of $P \leq .05$.

\section{Results}

None of all constructs had any leakage through the incision line. In group CC, leakage through one or more suture holes were present in half of the constructs $(\mathrm{p}=.027)$ (Fig. 1). No leakage was observed in any construct of RC, TPP and TC groups. (Table 1, Fig. 2).

\section{Discussion}

In this study, conventional cutting needles created significantly more leaks through the suture holes compared with the other types of needles used for the closure of enterotomies. In our study, enterotomy closure was performed using a 4-0 polydioxanone suture, a suture type commonly used for the closure of enterotomy incisions (Fossum and Hedlund, 2003; Brown, 2012).

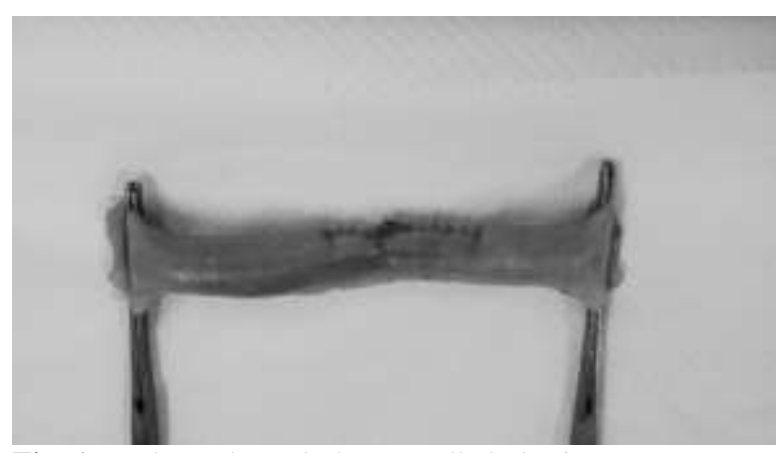

Fig. 1. Leakage through three needle holes in an enterotomy closed using a 4-0 polydioxanone simple interrupted suture armed in a conventional cutting swaged-on needle by infusion of methylene blue solution into the jejunal lumen. The jejunal segment is occluded by a pair of Doyen intestinal forceps.

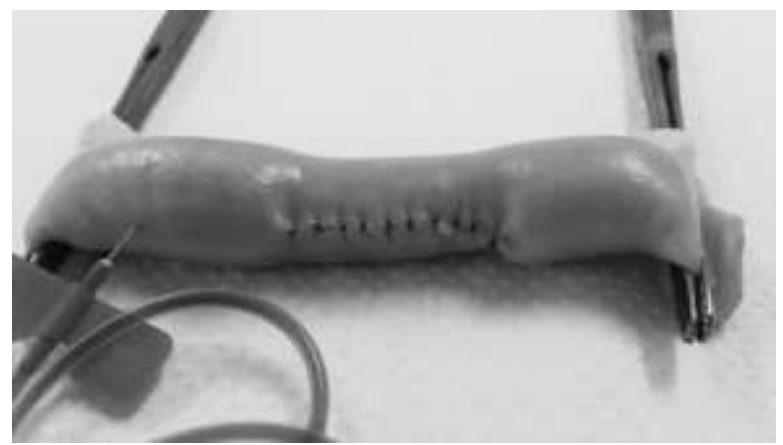

Fig. 2. Leakage testing in an enterotomy closed using a 4-0 polydioxanone simple interrupted suture armed in a taper cutting swaged-on needle by infusion of methylene blue solution into the jejunal lumen. The jejunal segment is occluded by a pair of Doyen intestinal forceps. No leakage was observed.

Table 1. Comparison of leakage holes created by 4 needle types used for closure of enterotomy incisions in dogs.

\begin{tabular}{lcccc}
\hline Groups & $\begin{array}{c}\text { No } \\
\text { leakage }\end{array}$ & Leakage & $\begin{array}{c}\text { Total number } \\
\text { of sections }\end{array}$ & P-value \\
\hline CC & 3 & 3 & 6 & 0.027 \\
RC & 6 & 0 & 6 & $>0.05$ \\
TPP & 6 & 0 & 6 & $>0.05$ \\
TCP & 6 & 0 & 6 & $>0.05$ \\
Control & 6 & 0 & 6 & $>0.05$ \\
$\begin{array}{l}\text { Total number } \\
\text { of sections }\end{array}$ & 27 & 3 & 30 & \\
\hline (CC): Conventional
\end{tabular}

(TPP): Taper point plus needle; (TCP): Taper-cutting needle.

The enterotomy was closed longitudinally in a simple interrupted full-thickness approximating pattern (Fossum and Hedlund, 2003; Shales et al., 2005; Brown, 2012; Ellison, 2014). This closure technique is commonly used by veterinarians.

Dehiscence of the enterotomy and leakage of intestinal contents into the peritoneal cavity, leading to septic peritonitis, is the most common and serious 
complication following intestinal surgery (Allen et al., 1992; Wylie and Hosgood, 1994; Weisman et al., 1999; Shales et al., 2005).

A poor surgical technique, hypoalbuminaemia, the presence of foreign bodies, preoperative peritonitis, inflammatory bowel disease, hypotension, or large intestinal surgery are the factors associated with the development of intestinal dehiscence (Baines, 2000; Ralphs et al., 2003; Ellison, 2014; Snowdon et al., 2016).

Leakage can develop between sutures of an enterotomy incision or around the needle holes created by needle passage through the intestinal wall, especially in a poorly viable intestine (Trier, 1979; Fossum and Hedlund, 2003). We did not notice any leakage between enterotomy sutures in the present study. Even if the enterotomy is securely closed, leakage through suture holes is still possible. In case of leakage, it might be difficult to differentiate between leakage from the incision line, suture holes or the combination of both (Thomson, 2007). In small animal intestinal surgery, taper point or taper cut needles are recommended because they are believed to create less trauma during passage through the intestinal wall (Weisman et al., 1999; Baines, 2000; Coolman et al., 2000; Ellison, 2014).

Following completion of the enterotomy closure, leakage is often tested by occluding the intestine proximal and distal to the incision side with the use of Doyen intestinal forceps or digital occlusion and infusion of saline into the intestinal lumen.

In a recent experimental study, $10 \mathrm{~cm}$ jejunal segments obtained from 38 dogs following a closed intestinal biopsy, saline volumes of $16.3-19 \mathrm{~mL}$ with digital occlusion and 12.1-14.8 mL with Doyen intestinal forceps occlusion were used to create an intraluminal pressure of $34 \mathrm{~cm}$ water during leak testing (Saile et al., 2010).

In the present study, a volume of $13.5 \mathrm{~mL}$ of diluted methylene blue solution was used to test for leakage in $10 \mathrm{~cm}$ jejunal segments after occlusion with Doyen intestinal forceps. We believe that with the present dye solution volume, an adequate intraluminal pressure of $34 \mathrm{~cm}$ water was achieved for leak testing (Saile et al., 2010). Methylene blue dye was used to facilitate visualization of leaks through the suture holes created during needle passage.

In the present study, constructs closed with conventional cutting needles, which were finer than the reverse cutting needles, showed significantly more leakage at the suture holes than in constructs belonging to the other groups.

This may be due to the fact that this type of needle, having a triangular cross-sectional area and a cutting surface on the needle inside a concave curvature, can produce a triangular hole during passage as the inside cutting edge cuts toward the incision (Trier, 1979; Dunn, 2005; Schmiedt, 2012). This already problematic triangular hole may become larger because of the force applied by the surgeon in our study to pass the needle through the intestinal wall. The tension applied to the suture may also distort and cut through the existing hole, thereby increasing the risk of leakage (Thomson, 2007; Schmiedt, 2012).

In the present study, constructs closed with a reverse cutting needle exhibited no leakage. This finding may be attributed to the different cutting surface configuration in the outer convex surface of the reverse cutting needle, creating a triangular hole that has the flat edge parallel to the edges of the incision (Dunn, 2005; Schmiedt, 2012).

The risk of hole distortion and tissue cut during needle passage and suture tension seems to be less with reverse cutting needles. The constructs of group TPP also showed no leakage holes. This finding may be due to the fact that this type of needle has an oval, instead of a conventional round cross-sectional, shape just behind its point, allowing the secure penetration and spreading of intestinal tissue without cutting through during needle passage (Dunn, 2005).

In this study, the TC group also showed no leakage holes. Taper cut needle is a combination of a reverse cutting point with a taper point, facilitating a smoother passage with less risk of cutting through the needle hole (Dunn, 2005; Schmiedt, 2012).

In a recent study it was found that the needle-to-suture ratio plays an important role in reducing needle hole bleeding in vascular anastomoses (Sergeant et al., 2016). Needle-to-suture ratio may impact leakage hole creation in intestinal surgery as well but this was not investigated in the study presented here.

This study has several limitations. It was performed in healthy cadaveric jejunum and was not evaluated in diseased intestinal tissues of living animals. Leakage in living animals might be less due to muscle contraction and clot formation around the needle hole. Small intestinal distensibility may also be different in such situations because of the ischemic and inflamed intestinal wall.

The large intestine was not evaluated in this study, as the findings related to leakage holes could be different. Further studies on living animals with intestinal disease should be conducted.

From the findings of the study presented here, conventional cutting needles created leakage holes in cadaveric healthy intestines during needle passage for closing an enterotomy incision. Reverse cutting, taper point, and taper cutting needles did not create leaking holes in the intestinal wall. Conventional cutting needles are contra-indicated to suture small intestines.

\section{Conflict of interest}

The authors declare that there is no conflict of interest. 


\section{References}

Allen, D.A., Smeak, D.D. and Schertel, E.R. 1992. Prevalence of small intestinal dehiscence and associated clinical factors: a retrospective study of 121 dogs. J. Am. Anim. Hosp. Assoc. 28, 70-76.

Baines, S. 2000. Small intestinal surgery 1. Principles. In Practice 22, 502-517.

Bellenger, C.R. 1982. Sutures. Part II. The use of sutures and alternative methods of closure. Compend. Contin. Educ. Pract. Vet. 4, 587-599.

Brown, D.C. 2012. Small intestine. In Veterinary Surgery Small Animal. Eds., Tobias, K.M. and Johnston, S.A. $1^{\text {st }}$ ed. St Louis, MO: Elsevier, pp: 1513-1541.

Coolman, B.R., Ehrhart, N., Pijanowski, G., Ehrhart, E.J. and Coolman, S.L. 2000. Comparison of skin staples with sutures for anastomosis of the small intestine in dogs. Vet. Surg. 29, 293-302.

Dunn, D.L. 2005. The surgical needle. In Ethicon Wound Closure Manual. Ed., Dunn, D.L. Somerville, N.J.: Ethicon, pp: 42-54.

Ellison, G.W. 2014. Enterotomy, In Current Techniques in Small Animal Surgery. Ed., Bojrab, M, J. $5^{\text {th }}$ ed. Jackson, WY: Teton New Media, pp: 276-280.

Fossum, T.W. and Hedlund, C.S. 2003. Gastric and intestinal surgery. Vet. Clin. North Am. Small Anim. Pract. 33, 1117-1145.

Ralphs, S.C., Jessen, C.R. and Lipowitz, A.J. 2003. Risk factors for leakage following intestinal anastomosis in dogs and cats: 115 cases (19912000). J. Am. Vet. Med. Assoc. 223, 73-77.

Saile, K., Boothe, H.W. and Boothe, D.M. 2010. Saline volume necessary to achieve predetermined intraluminal pressures during leak testing of small intestinal biopsy sites in the dog. Vet. Surg. 39, 900903.
Schmiedt, C.W. 2012. Suture material, tissue staplers, ligation devices and closure methods. In Veterinary Surgery Small Animal. Eds., Tobias, K.M. and Johnston, S.A. $1^{\text {st }}$ ed. St Louis, MO: Elsevier, pp: 187-200.

Schwartz, Z. and Coolman, B.R. 2018. Disposable skin staples for closure of linear gastrointestinal incisions in dogs. Vet. Surg. 47, 285-292.

Sergeant, P., Kocharian, R., Patel, B., Pfefferkorn, M. and Matonick. J. 2016. Needle-to-suture ratio, as well as suture material, impacts needle-hole bleeding in vascular anastomoses. Interact. Cardiovasc. Thorac. Surg. 22, 813-816.

Shales, C.J., Warren, J., Anderson, D.M., Baines, S.J. and White, R.A. 2005. Complications following full-thickness small intestinal biopsy in 66 dogs: a retrospective study. J. Small Anim. Pract. 46, 317321.

Snowdon, K.A., Smeak, D.D. and Chiang, S. 2016. Risk factors for dehiscence of stapled functional end-to end intestinal anastomosis in dogs: 53 cases (2001-2012). Vet. Surg. 45, 91-99.

Thomson, G.A. 2007. An investigation of leakage tracts along stressed suture lines in phantom tissue. Med. Eng. Phys. 29, 1030-1034.

Trier, W.C. 1979. Considerations in the choice of surgical needles. Surg. Gynecol. Obstet. 149(1), 8494.

Weisman, D.L., Smeak, D.D., Birchard, S.J. and Zweigart, S.L. 1999. Comparison of a continuous suture pattern with a simple interrupted pattern for enteric closure in dogs and cats: 83 cases (19911997). J. Am. Vet. Med. Assoc. 214, 1507-1510.

Wylie, K.B. and Hosgood, G. 1994. Mortality and morbitidy of small and large intestinal surgery in dogs and cats: 74 cases (1980-1992). J. Am. Anim. Hosp. Assoc. 30, 469-474. 